\title{
NFATC2 is a novel therapeutic target for colorectal cancer stem cells
}

This article was published in the following Dove Press journal:

OncoTargets and Therapy

Tingyuan Lang,' Xiaojuan

Ding,' Liangsheng Kong,'

Xiaoyan Zhou,' Zhiqi

Zhang, ${ }^{2}$ Huangxian Ju, ${ }^{1,3}$

Shijia Ding'

'Key Laboratory of Laboratory Medical Diagnostics (Ministry of Education of China), Department of Laboratory Medicine, Chongqing Medical University, Chongqing 4000 I6, People's Republic of China; ${ }^{2}$ Department of General Surgery, Shanghai Jiao Tong University Affiliated Sixth People's Hospital, Shanghai 200233, People's Republic of China; ${ }^{3}$ State Key Laboratory of Analytical Chemistry for Life Science, School of Chemistry and Chemical Engineering, Nanjing University, Nanjing 210023 ,

People's Republic of China
Correspondence: Shijia Ding

Key Laboratory of Clinical Laboratory Diagnostics (Ministry of Education),

College of Laboratory Medicine,

Chongqing Medical University,

No. I, Yixueyuan Road, Yuzhong

District, Chongqing 4000I6,

People's Republic of China

Tel +86 I33 20335509

Fax +8602368485786

Email dingshijia@163.com

Huangxian Ju

State Key Laboratory of Analytical Chemistry for Life Science, School of

Chemistry and Chemical Engineering,

Nanjing University, No. 163, Xianlin

Road, Qixia District, Nanjing 210023,

People's Republic of China

Tel +86 I30 $0342 \quad 1236$

Fax +860258968 776I

Email hxju@nju.edu.cn
Background: Colorectal cancer stem cells (CRC-SCs) contribute to the initiation and progression of colorectal cancer (CRC). However, the underlying mechanisms for the propagation of CRC-SCs have remained elusive.

Purpose: The objective of this study was to study the role of NFATC2 in maintenance of the stemness in CRC-SCs.

Method: The expression levels of mRNA and protein were determined by qRT-PCR and western-blot, respectively. CRC-SCs were isolated by spheroid formation assay and flowcytometry. The sphere-forming and self-renewal abilities of CRC-SCs were determined by spheroid formation assay. The tumorigenicity of CRC-SCs was determined by cell-derived xenograft model. Gene manipulation was performed by lentivirus-mediated delivery system.

Results: We first found that NFATC2 is upregulated in primary CRC-SCs. Overexpression of NFATC2 promotes self-renewal and the expression of stem cell markers of CRC-SCs. Conversely, knockdown of NFATC2 attenuates stem cell-like properties of CRC-SCs. Mechanistic analysis indicated that NFATC2 upregulates the expression of AJUBA, downregulates the phosphorylation level of YAP, and therefore activates the transcriptional activities of YAP and promotes the stemness of CRC-SCs.

Conclusion: Our findings demonstrate NFATC2 as an oncogene that can promote the stemness of CRC-SCs. This work suggests a novel therapeutic strategy against CRC caused by aberrant expression of NFATC2.

Keywords: NFATC2, colorectal cancer, stem cells, stemness, YAP

\section{Introduction}

Colorectal cancer (CRC) is the leading cause of cancer death. ${ }^{1}$ Although current therapeutic strategies have reduced mortality, limited options are available for recurrence and resistance. ${ }^{2}$ Cancer stem cells (CSCs) are a small subset of cancer cells capable of self-renewal and differentiating. ${ }^{3} \mathrm{CSC}$ s are responsible for tumor initiation and progression, resistant to chemotherapy and radiation, and thus have been recognized as a central hypothesis for the failure of cancer treatment. ${ }^{4} \mathrm{CSC}$ s have been identified in almost all solid tumors including prostate, ovary, breast as well as colorectal cancers. ${ }^{5-8}$ Identification of oncogenes and signaling pathways sustaining the properties of CSCs is critical for the development of an effective therapeutic strategy against CSCs. ${ }^{9,10}$

NFATC2 is a member of the nuclear factor of activated T-cell (NFAT) family which has well documented roles in the immune system. ${ }^{11}$ However, its functions in cancer initiation and progression remained elusive. It has been reported that NFATC2 negatively regulates the promoter of cyclin-dependent kinase $4^{12}$ and NFATC2 knockout mouse exhibits altered cell cycle control, ${ }^{13}$ indicating a major role of NFATC2 in regulating the cell cycle. NFATC2 activates the mdm 2 oncogene independent of 
$\mathrm{P} 53^{14}$ and enhances the metastasis of breast cancer cell by the upregulation of cyclooxygenase- 2 and prostaglandins. ${ }^{15}$ In addition, NFATC 2 upregulates the mRNA level of human telomerase reverse transcriptase in activated peripheral blood lymphocytes. ${ }^{16}$ Moreover, forced activation of NFATC2 activates c-Myc, ${ }^{17}$ an important gene for the maintenance of stem cells, which suggests the potential role of NFATC2 in CSCs. Further efforts are still needed to explore the biological function of NFATC2 in CSCs.

Hippo is a highly conserved signaling cascade that regulates organ size and cell fate. ${ }^{18}$ The core kinase cascade of Hippo pathway includes mammalian STE20-like protein kinase (MST1/2) and large tumor suppressor homolog (LATS1/2). In active state, Hippo signaling phosphorylates transcriptional co-activator YAP and results in its $\beta$-transducin repeat-containing E3 ubiquitin protein ligase ( $\beta$-TRCP)dependent degradation. ${ }^{19}$ YAP regulates the expression of genes involved in key cellular functions, such as cell cycle, apoptosis, as well as maintenance of stem cell pluripotency. ${ }^{20}$ Thus, YAP inhibition limits the organ size. Hippo/YAP axis is an important target for colorectal cancer therapy, and several small molecules for targeting various molecular components of Hippo/YAP signaling pathway have been developed. ${ }^{21}$

In this study, we reported that NFATC2 promotes the stemness of CRC-SCs through AJUBA-mediated YAP activation. Our work thus demonstrates that NFATC2 is a novel therapeutic target for colorectal cancer stem cells (CRC-SCs) and suggests that YAP inhibitor could be an effective therapeutic strategy against $\mathrm{CRC}$ caused by aberrant expression of NFATC2.

\section{Materials and methods Cell culture and transfection}

Human cancer cell lines (HCT-116, HT-29) were purchased from the American Type Culture Collection. Lenti-X 293T cell line was purchased from Clontech Laboratories (Palo Alto, CA, USA). Cell lines were cultured in DMEM with added fetal bovine serum, glutamine, penicillin, and streptomycin. The absence of mycoplasma contamination was confirmed using Myco Alert (Lonza, Basel, Switzerland).

\section{Clinical samples}

The tumor specimens were obtained from CRC patients who underwent surgery at the Department of Surgery at Shanghai Jiao Tong University-Affiliated Sixth People's Hospital East Campus. This study was approved by Institutional Reviewing Board of Shanghai Jiao Tong University-Affiliated Sixth People's Hospital East Campus. Written informed consent was obtained from each patient.

\section{In vivo xenograft}

All animal experiments were approved by the Institutional Animal Care and Use Committee at Chongqing Medical University. The experimental procedures all complied with the National Institutes of Health's Guide for the Care and Use of Laboratory Animals; all guidelines for the welfare of the animals were followed. ${ }^{22}$ Female nude mice have been injected with CRC sphere-derived cells. Kinetic of tumor formation was evaluated every 5 days.

\section{Plasmids, primers, short hairpin RNAs (shRNAs), and reagents}

Full-length cDNA of NFATC2 were generated by reversetranscription PCR and cloned into $\mathrm{pCDH}$ expression vector. For shRNA experiments, two independent shRNAs against specific genes were cloned into the PLKO.1 vector. Primers are listed in Table S1, shRNAs are listed in Table S2, and antibodies are listed in Table S3. All common reagents were purchased from Sigma-Aldrich Co., St Louis, MO, USA.

\section{Lentiviral generation and infection}

Lenti-X Packaging Single Shots (Clontech Laboratories Inc.) were used to create lentiviruses. The cells were infected with media with lentivirus particles. Stable cell line was selected by puromycin for 10 days. The efficiency of knockdown and overexpression was determined by immunoblot.

\section{Spheroid formation assay}

The primary cells were isolated from tumor tissues and maintained in StemPro hESC SFM (Thermo Fisher Scientific, Waltham, MA, USA) supplemented with $8 \mathrm{ng} \mathrm{mL}^{-1}$ basic fibroblast growth factor (Sigma-Aldrich Co.) in ultra-low attachment six-well plates (Corning Incorporated, Corning, NY, USA). The cells were seeded at a density of $1 \times 10^{3}$ cells per well into ultra-low attachment six-well plates (Corning Incorporated). After 10-15 days of culture, the spheroids were observed under a phase-contract microscopy (Leica Microsystems, Wetzlar, Germany).

\section{Western blot}

The protein samples were separated by sodium dodecyl sulfatepolyacrylamide gel electrophoresis and blotted onto immobilon-P membranes (EMD Millipore, Billerica, MA, USA) followed by incubation with the primary antibodies at $4{ }^{\circ} \mathrm{C}$ overnight. After incubation with the relevant horseradish peroxidaseconjugated secondary antibody and Clarity ${ }^{\mathrm{TM}}$ Western ECL Substrate (Bio-Rad Laboratories Inc., Hercules, CA, USA), signals were visualized with the ChemiDoc MP system (BioRad). Full scans of the blots are shown in Supplementary materials. 


\section{Immunofluorescence analysis}

The cells or spheroids were rinsed and fixed using 4\% paraformaldehyde for 10 minutes at room temperature. Antigen retrieval was performed by incubation with the antigen retrieval buffer $(100 \mathrm{mM}$ Tris, $5 \%$ urea, $\mathrm{pH} 9.5)$ at $95^{\circ} \mathrm{C}$ for 10 minutes. Cells were then permeabilized with PBS containing 0.1 Triton X-100 for 15 minutes. After blocking with $1 \%$ bovine serum albumin and $22.52 \mathrm{mg} / \mathrm{mL}$ glycine in PBS+ $0.1 \%$ Tween 20 for 30 minutes, cells were incubated with the primary antibody at $4{ }^{\circ} \mathrm{C}$ overnight and then with the secondary antibody conjugated to DyLight 488, Alexa 488 or 555 (Abcam; Cambridge, MA, USA) for 1 hour at room temperature. Cells were examined using a total internal reflection fluorescence (TIRF) microscopy (Leica Microsystems, Wetzlar, Germany). Leica Application Suite X software (Leica Microsystems) was used to obtain the image.

\section{Quantitative real-time reverse- transcription PCR}

Total RNA was isolated with an RNeasy Mini Kit (Qiagen NV, Venlo, the Netherlands). cDNA was synthesized by SuperScript II reverse transcriptase (Thermo Fisher Scientific). The cDNA products were then subjected to TaqMan gene expression assay using specific primers. Amplification data were collected by ABI Prism 7000HT Real-Time PCR system. The results were calculated using the comparative threshold cycle $\left(\mathrm{C}_{\mathrm{t}}\right)$ method with GAPDH as a control.

\section{Statistical analysis}

Each experiment was repeated for at least three times. Data are expressed as the mean $\pm \mathrm{SD}$. The significance of the data between experimental groups was determined by Student's $t$-test (unpaired, two-tailed), ${ }^{*} P<0.05, * * P<0.01, * * * P<0.001$.

\section{Results}

\section{NFATC2 is upregulated in CRC-SCs}

Sphere formation is recognized as the most prominent characteristic of CRC-SCs and can be used for in vitro study of CRC-SC function. ${ }^{23}$ To investigate whether the expression level of NFATC2 is dysregulated in CRC-SCs, we first isolated the primary $\mathrm{CRC}$ spheres from tumor tissue of CRC patients by suspension culture. ${ }^{24-26}$ The mRNA and protein level of NFATC2 in the spheres and adherent cells were then analyzed by quantitative real-time polymerase chain reaction (qRT-PCR) and Western blot, respectively. As shown in Figure 1A and B, compared with adherent cells, NFATC 2 expression was upregulated in CRC spheres. While, the expression level of NFATC2 was recovered to the level of origin when the spheres were reattached (Figure 1A and B). This result indicated that NFATC2 is upregulated in CRC-SCs. Next, as CD44 and CD133 are well-identified CRC-SC markers, ${ }^{27,28}$ to confirm this finding, we enriched CRC-SCs by flow cytometry sorting ${ }^{24-26}$ and examined the mRNA level of NFATC2 in CD44 ${ }^{+}$and CD133 ${ }^{+}$ primary CRC cells and negative control cells. As shown in Figure 1C, the NFATC2 expression was upregulated in sorted $\mathrm{CD} 44^{+}$or $\mathrm{CD} 133^{+}$primary CRC cells, which confirmed that NFATC2 is upregulated in CRC-SCs. Furthermore, we examined the mRNA level of NFATC2, CD44, and CD133 in primary CRC spheres by qRT-PCR, and Spearman correlation analysis was subsequently performed. The results showed that the expression level of NFATC2 was positively correlated with the expression level of CD44 or CD133 in primary CRC spheres (Figure 1D). These data demonstrated that NFATC2 was upregulated in CRC-SCs.

\section{NFATC2 promotes CRC-SC properties of CRC cells}

To further investigate the functional role of NFATC2 in CRC-SCs, we overexpressed NFATC2 in primary CRC cells by lentivirus delivery system (Figure S1). As shown in Figure 2A, forced expression of NFATC2 in primary CRC cells significantly increased the number and size of the spheres, which indicated that NFATC2 promotes the sphere formation capacity of primary CRC cells. In addition, we examined the number of 1 st, 2nd, and 3rd passaged spheres and found that NFATC2 promotes self-renewal capacity of primary CRC cells on serial passage (Figure 2B). Moreover, NFATC2 overexpression increased the expression of CSC-SC markers (CD44 and CD133) as indicated by qRT-PCR (Figure 2C) and Western blot (Figure 2D). These results demonstrated that NFATC2 promotes CRC-SC properties of CRC cells.

\section{NFATC2 is required for the maintenance of CRC-SCs}

To further confirm the critical role of NFATC2 in the maintenance of CRC-SCs, we knocked down NFATC2 in primary CRC cells by two independent lentivirus-based shRNAs (Figure S2). As shown in Figure 3A and B, knockdown of NFATC2 attenuates sphere-forming and self-renewal abilities of primary CRC cells. In addition, NFATC2-knockdown primary CRC cells exhibited decreased expression levels of CD44 and CD133 as identified by qRT-PCR and Western blot (Figure 3C and D). Furthermore, we examined the effect of NFATC depletion on tumorigenesis of primary CRC 

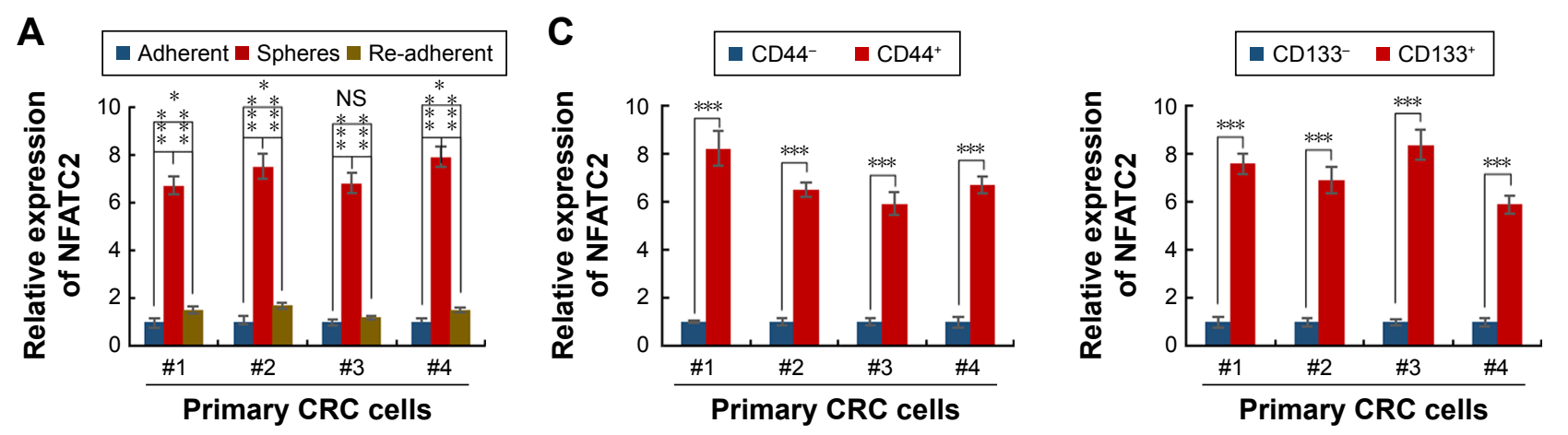

B
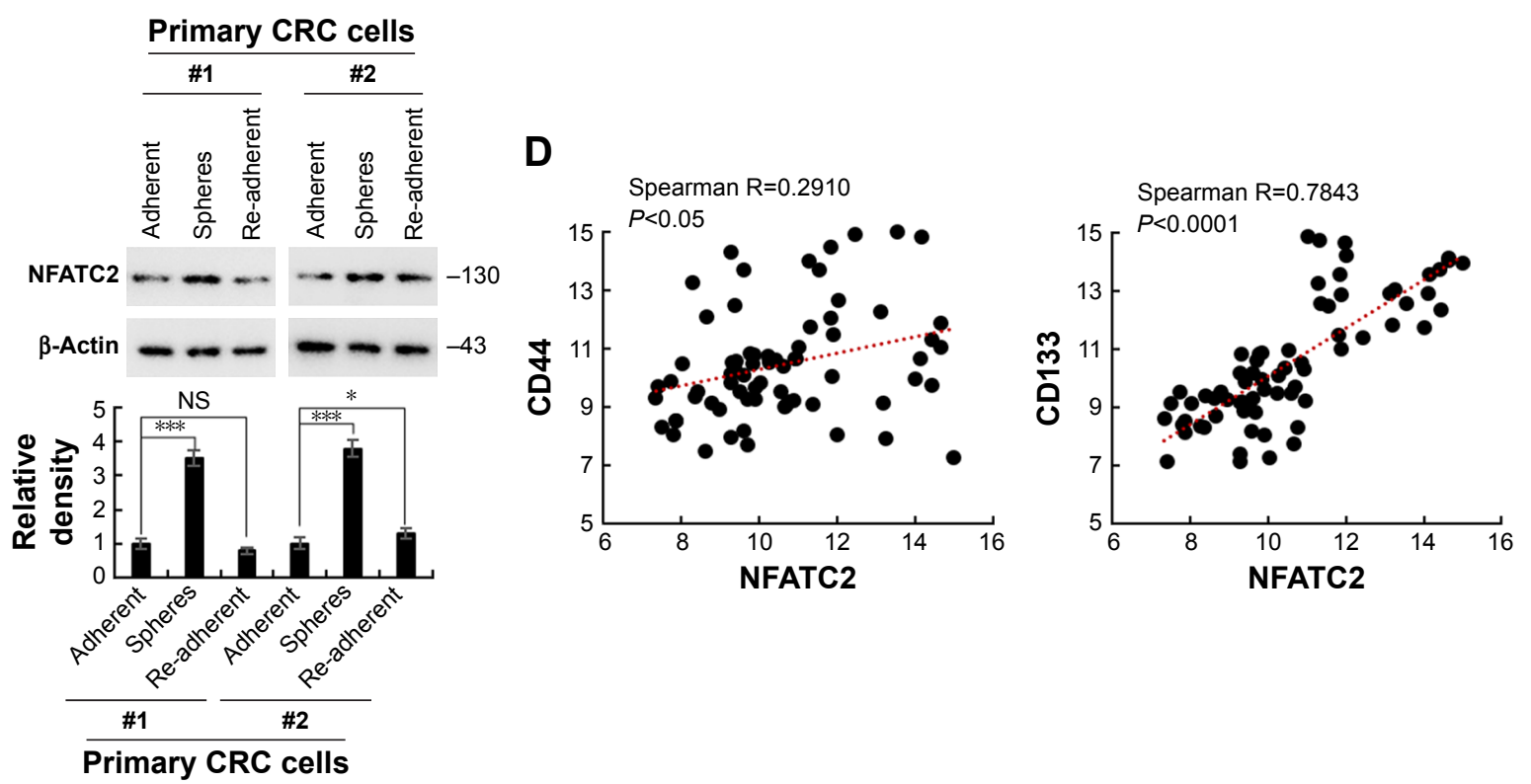

Figure I NFATC2 is upregulated in CRC-SCs. (A) qRT-PCR analysis of NFATC2 in primary CRC spheres and re-adherent cells relative to adherent cells. Primary CRC cells were isolated from the cancer tissues of CRC patients (No I, 2, 3, and 4). Spheres were obtained by suspension culture. (B) Western blot analysis of NFATC2 in primary CRC spheres and adherent and re-adherent cells. (C) qRT-PCR analysis of NFATC2 in sorted CD44+ (left) or CDI33+ (right) primary CRC cells relative to negative cells. Primary CRC cells were isolated from the cancer tissues of colorectal cancer patients (No I, 2, 3, and 4). CD $44^{+}$or CDI33 cells were obtained by flow cytometry. (D) The correlation between the transcription level of NFATC2 and CD44 (left) and CDI33 (right) in pin primary CRC sphere-derived cells. The mRNA level of each gene was determined by $\mathrm{qRT}$-PCR. Data were normalized to GAPDH as $\triangle \mathrm{CT}$ and analyzed by Spearman's correlation analysis. Data are represented as mean \pm SD; $* P<0.05$, $* * * P<0.00$ I; two-tailed Student's t-test.

Abbreviations: CRC, colorectal cancer; NFATC2, nuclear factor of activated T-cells, cytoplasmic 2; CRC-SCs, colorectal cancer stem cells; qRT-PCR, quantitative real-time polymerase chain reaction.

cells in vivo and found that NFATC depletion significantly decreased tumorigenesis of CRC cells in vivo. These results demonstrated that NFATC2 is required for the maintenance of the stemness of CRC-SCs.

\section{YAP activity is necessary for NFATC2- mediated CRC-SC properties}

To reveal the underlying mechanism of NFATC2-mediated CRC-SC properties, NFATC2-overexpressing and knockdown HCT-116 and HT-29 stable cell lines (Figure S3A and B) were generated and qRT-PCR array was performed to investigate the regulatory effect of NFATC2 on several signaling pathways which were recognized to be important for the maintenance of the properties of CRC-SCs, such as $\mathrm{Wnt}^{29}$ and Hippo/YAP ${ }^{18-20}$ signaling pathway. As shown in Figure S3C, overexpression of NFATC2 significantly upregulated the expression levels of the target genes of Hippo/ YAP signaling pathway (CTGF and Gli2) ${ }^{18-20}$ in both HCT116 and HT-29 cells. Conversely, reduced mRNA expression levels of CTGF and Gli2 were observed in NFATC2-knockdown HCT-116 and HT-29 cells (Figure S3D). These results indicated that Hippo/YAP signaling might be the downstream effector of NFATC2.

To confirm this finding, we next depleted YAP in NFATC2-overexpressing primary CRC cells (Figure S3E) to investigate the role of YAP activity in NFATC2-induced CRC-SC properties. As shown in Figure 4A and B, knockdown of YAP abolished the stimulatory effect of NFATC2 
A
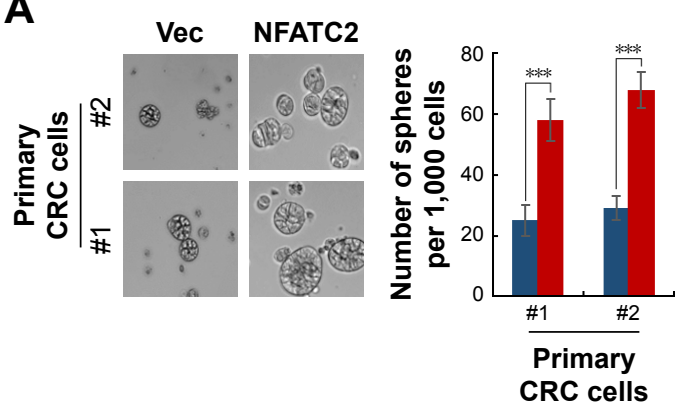

B

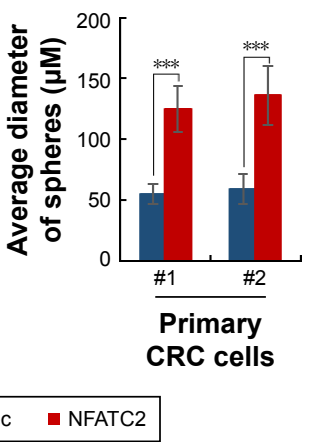

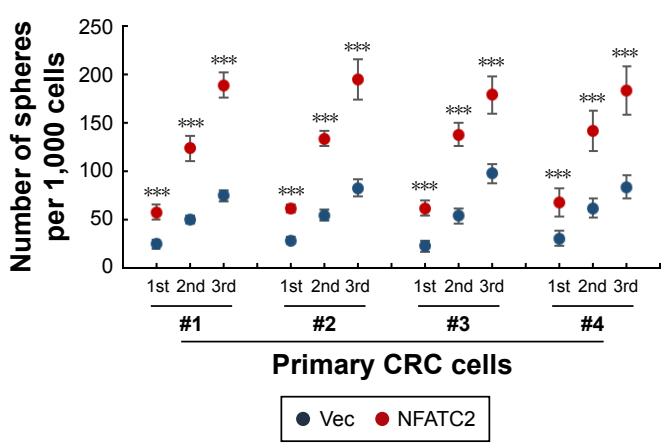
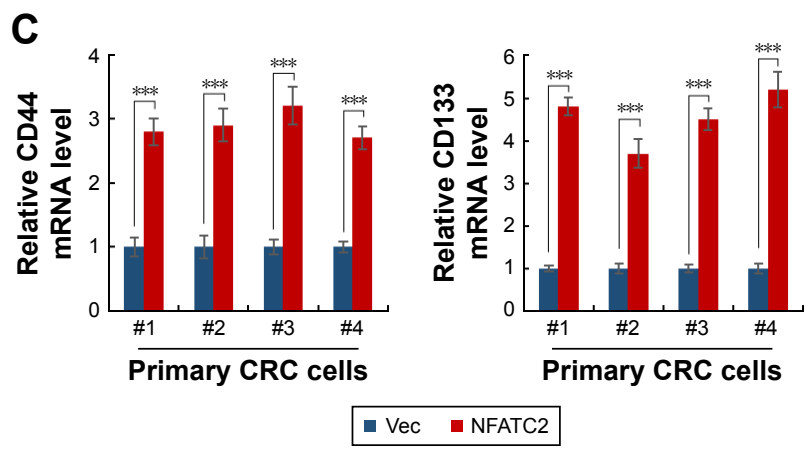

D
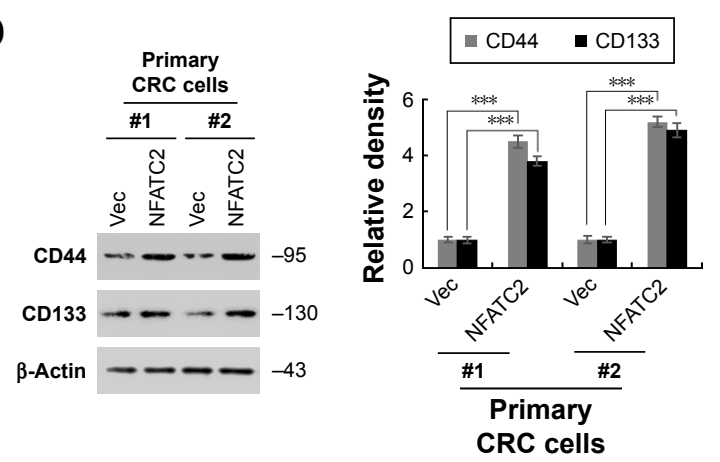

Figure 2 Overexpression of NFATC2 promotes the stemness of CRC cells. (A, B) Sphere formation assay of NFATC2-overexpressing and control primary CRC cells. Primary CRC cells were isolated from the cancer tissues of CRC patients (No I and 2). The Ist passaged spheres were obtained by suspension culture for I5 days and the number and average diameters of the spheres were counted (A). The number of Ist, 2 nd, and 3rd passaged spheres isolated from the cancer tissues of CRC patients (No I, 2, 3, and 4) was also counted (B). (C) qRT-PCR analysis of CD44 (left) and CDI33 (right) in NFATC2-overexpressing and control primary CRC cells. Primary CRC cells were isolated from the cancer tissues of CRC patients (No I and 2). NFATC2-overexpressing and control cells were generated by lentivirus delivery system. (D) Western blot analysis of CD44 and CDI33 in NFATC2-overexpressing and control primary CRC cells. Data are represented as mean \pm SD; *** $P<0.00$ I; two-tailed Student's $t$-test. Abbreviations: CRC, colorectal cancer; NFATC2, nuclear factor of activated T-cells, cytoplasmic 2; CRC-SCs, colorectal cancer stem cells; qRT-PCR, quantitative real-time polymerase chain reaction.

on the sphere-forming and self-renewal abilities of CRC-SCs. In addition, YAP depletion recovered the increased expression levels of CD44 and CD133 in NFATC2-overexpressing primary CRC cells as indicated by qRT-PCR and Western blot (Figure 4C and D). Furthermore, YAP knockdown abolished the stimulatory effect of NFATC2 on tumorigenesis of primary CRC cells in vivo (Figure 4E), which indicated that YAP activity is critical for NFATC2 promoting the stemness of CRC-SCs.

The above results demonstrated that NFATC2 regulates Hippo/YAP signaling pathway in CRC cells and that YAP activity is critical for NFATC2-induced CRC-SC properties.

\section{NFATC2 enhances YAP activity by the upregulation of AJUBA}

To investigate the mechanism underlying the regulatory effect of NFATC2 on the activity of YAP, we first determined whether NFATC2 regulates YAP transcription. As shown in Figure S4A, there is no significant difference in YAP
mRNA level between NFATC2-overexpressing cells and control cells (Figure S4A). While, as shown in Figure 5A, NFATC2 decreased the phosphorylation level of YAP in HCT-116 and HT-29 cells. In Hippo/YAP signaling pathway, a kinase cascade was involved in MST1/2, and LATS1/2 phosphorylates YAP and results in its $\beta$-TRCP-dependent degradation. ${ }^{18-20}$ Thus, this result indicated that NFATC2 might have affected the stabilization of YAP. To confirm this, we measured the effect of NFATC2 on the stabilization of endogenous YAP in HCT-116 cells following inhibition of new protein synthesis by cycloheximide (CHX). Indeed, as shown in Figure 5B, overexpression of NFATC2 stabilized the endogenous YAP in HCT-116 cells. These results thus demonstrated that NFATC2 activates YAP by promoting its stabilization and suggested that NFATC2 may target the upstream regulators of YAP.

To further investigate the target of NFATC2 in Hippo/ YAP signaling pathway, we performed qRT-PCR array to study the effect of NFATC2 on the transcription of key components in Hippo/YAP signaling pathway. As shown in 
A

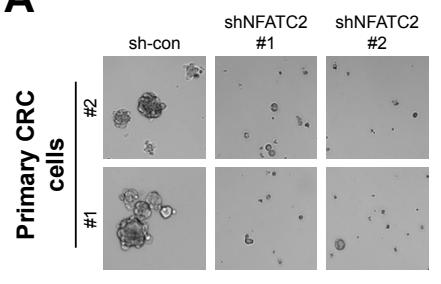

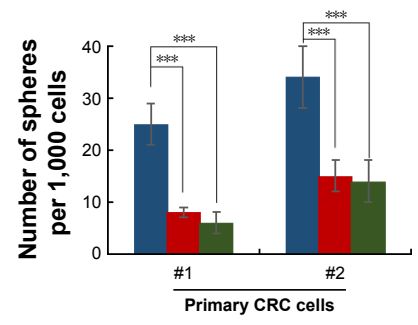

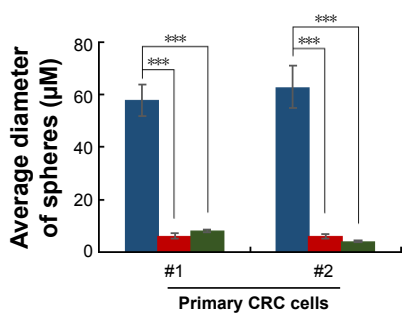

- sh-con $\mathbf{m}$ shNFATC2\#1
B

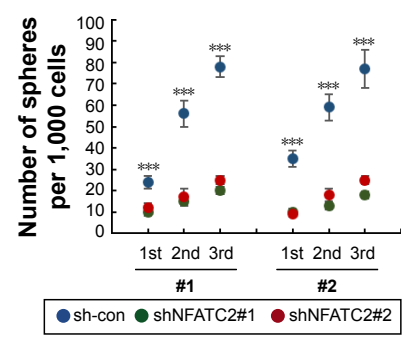

C
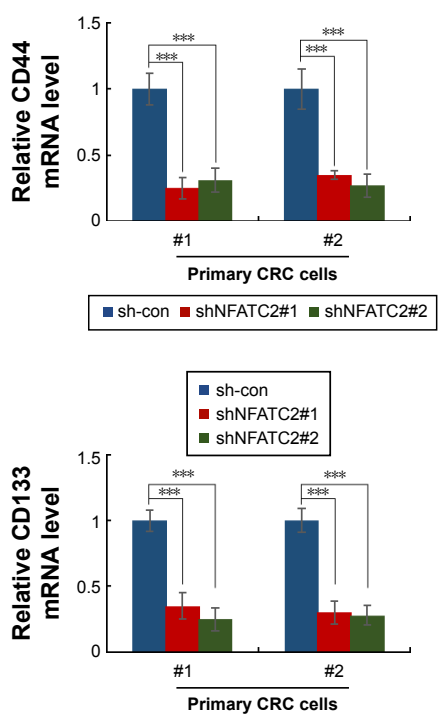

D
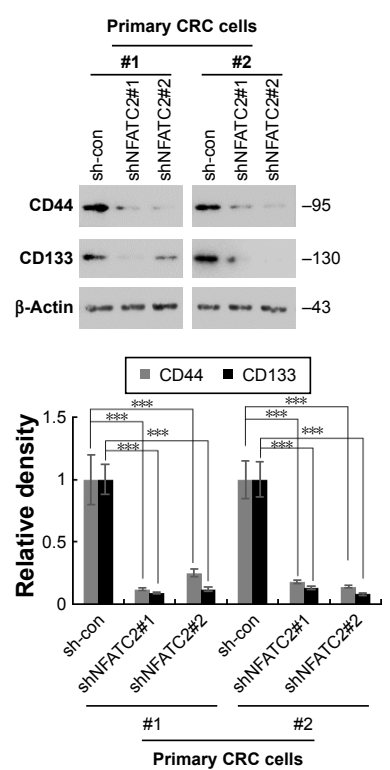

E
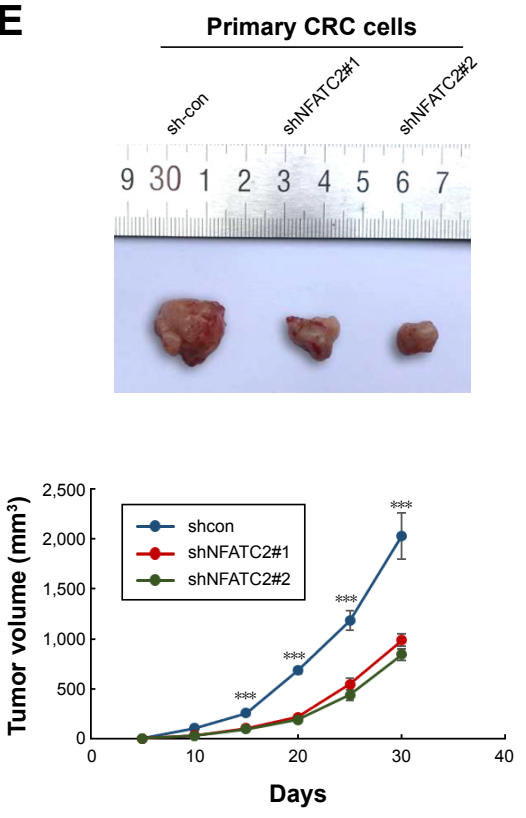

Figure 3 Knockdown of NFATC2 inhibits the stemness of CRC cells. (A, B) Sphere formation assay of NFATC2-knockdown and control primary CRC cells. Primary CRC cells were isolated from the cancer tissues of CRC patients (No I and 2). The Ist passaged spheres were obtained by suspension culture for I5 days and the number and average diameters of the spheres were counted (A). The number of Ist, 2nd, and 3rd passaged spheres was also counted (B). (C) qRT-PCR analysis of CD44 (top) and CDI 33 (bottom) in NFATC2-knockdown and control primary CRC cells. Primary CRC cells were isolated from the cancer tissues of CRC patients (No I and 2). NFATC2knockdown and control cells were generated by lentivirus delivery system. (D) Western blot analysis of CD44 and CDI33 in NFATC2-knockdown and control primary CRC cells. (E) Tumorigenesis of NFATC2-knockdown and control primary CRC cells. Data are represented as mean \pm SD; $* * * P<0.00$ I; two-tailed Student's $t$-test.

Abbreviations: CRC, colorectal cancer; NFATC2, nuclear factor of activated T-cells, cytoplasmic 2; CRC-SCs, colorectal cancer stem cells; qRT-PCR, quantitative real-time polymerase chain reaction.

Figure 5C, we found that NFATC2 significantly upregulates the mRNA level of AJUBA. As a fact that AJUBA activates YAP by inhibition of the kinase activity of LATS $1 / 2,{ }^{30}$ we postulate that AJUBA mediates the stimulatory effect of NFATC2 on YAP activity. To confirm this, AJUBA was knocked down in NFATC2-overexpressing HCT-116 and HT-29 cells (Figure S4B), and the effect of NFATC2 on YAP activity was measured in these cells. As shown in Figure 5D, knockdown of AJUBA abolished the stimulatory effect of NFATC2 on the expression of CTGF and GLI2, indicating the critical role of AJUBA in NFATC2-induced upregulation of YAP activity. Taken together, the above results demonstrated that NFATC2 enhances YAP activity by the upregulation of AJUBA.

\section{Discussion}

In this study, we reported that NFATC2 promotes the stemness of CRC-SCs through AJUBA-mediated activation of Hippo/YAP signaling (Figure 6).

Accumulating evidence indicates that cancer initiation and progression are caused primarily by CSCs, a small subset of cancer cells, that shares stem-like properties through epithelial-mesenchymal transition (EMT). ${ }^{31,32}$ CSCs also contribute to recurrence, metastasis as well as chemo and radio-resistance in cancer. ${ }^{1-4}$ Thus, CSCs are the main target of cancer therapy. Although efforts toward understanding CSC biology in past decades identified a set of oncogenes and related signaling pathways sustaining "stemness," still wide exploration is needed. 
A

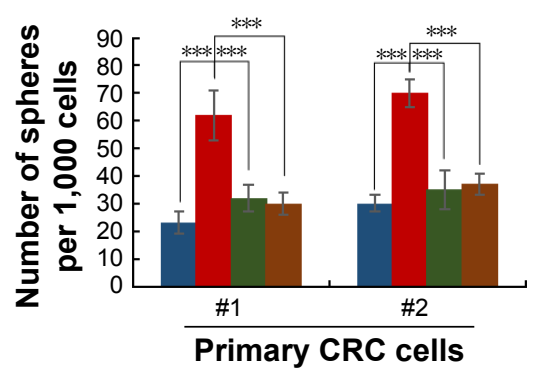

घec $\square$ NFATC2-sh-con $\square$ NFATC2-shYAP\#1 $\square$ NFATC2-shYAP\#2
D

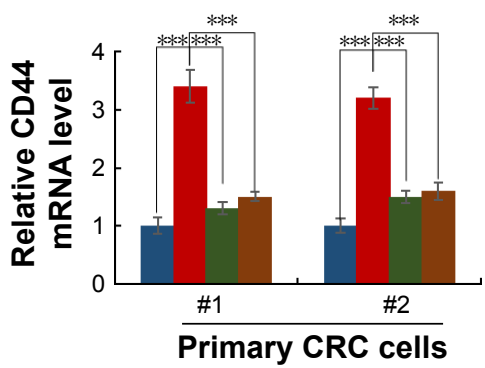

Primary CRC cells

\section{Primary CRC cells}

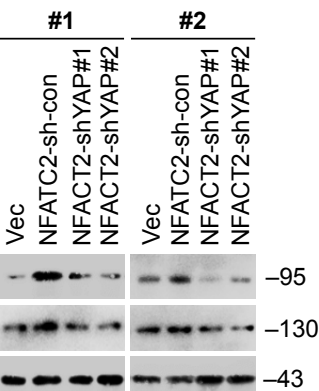

B
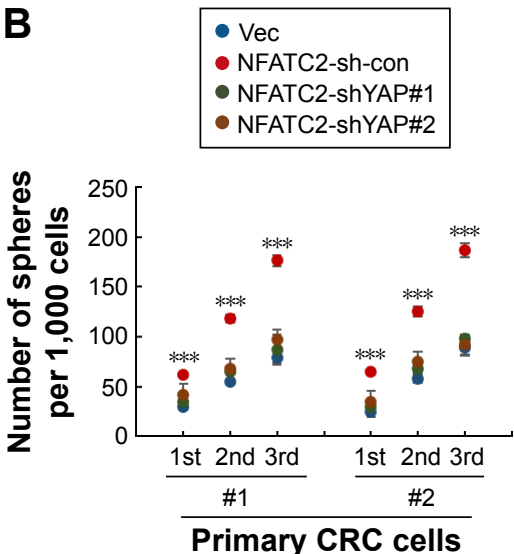

Primary CRC cells

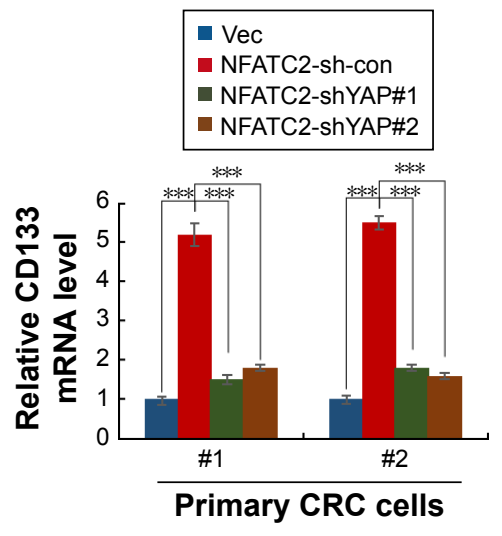

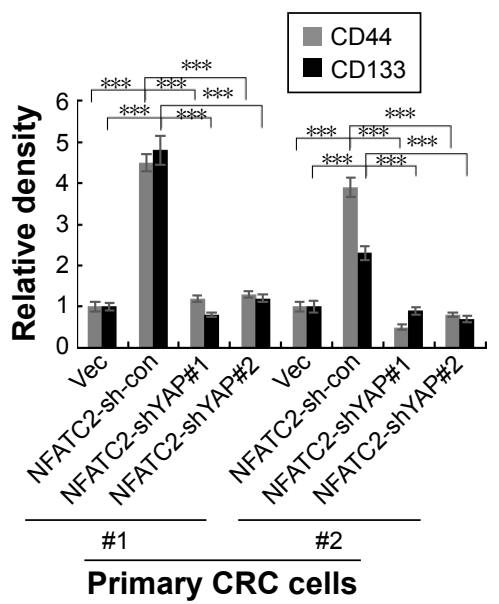

E

\section{Primary CRC cells}

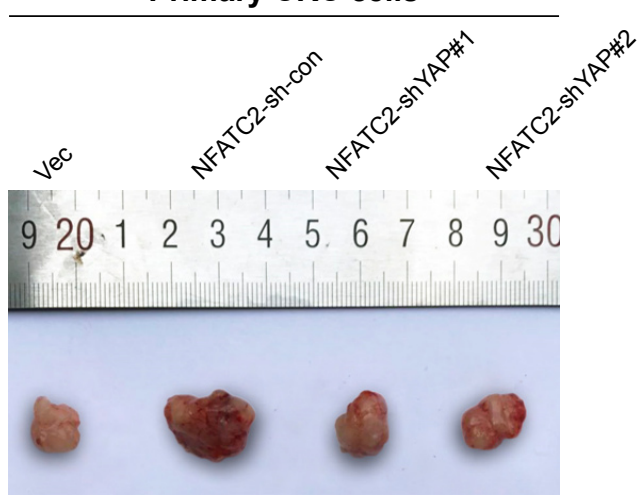

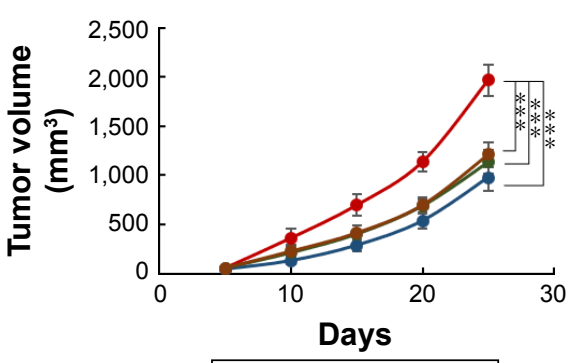

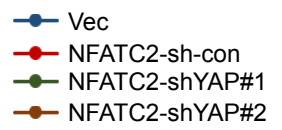

Figure 4 YAP activity is necessary for NFATC2 for maintaining the stemness in CRC cells. (A, B) Sphere formation assay of YAP-knockdown NFATC2-overexpressing primary CRC cells and control cells. Primary CRC cells were isolated from the cancer tissues of CRC patients (No I and 2). The Ist passaged spheres were obtained by suspension culture for 15 days and the number of the spheres were counted (A). The number of Ist, 2nd, and 3rd passaged spheres was also counted (B). (C) qRT-PCR analysis of CD44 (top) and CDI33 (bottom) in YAP-knockdown NFATC2-overexpressing primary CRC cells and control cells. Primary CRC cells were isolated from the cancer tissues of CRC patients (No I and 2). YAP-knockdown NFATC2-overexpressing primary CRC cells and control cells were generated by lentivirus delivery system. (D) Western blot analysis of CD44 and CDI33 in YAP-knockdown NFATC2-overexpressing primary CRC cells and control cells. (E) Tumorigenesis of YAP-knockdown NFATC2-overexpressing primary CRC cells and control cells. Data are represented as mean $\pm S D$; $* * * P<0.00$ I; two-tailed Student's $t$-test.

Abbreviations: CRC, colorectal cancer; NFATC2, nuclear factor of activated T-cells, cytoplasmic 2; CRC-SCs, colorectal cancer stem cells; qRT-PCR, quantitative real-time polymerase chain reaction.

NFATC2 is the first identified member of the NFAT family, and its roles in the immune system are studied well. ${ }^{11}$ Recent studies showed that there are potential functional roles of NFATC2 in the maintenance of CSCs. First, Zafari et al reported that the mRNA level of NFATC2 is upregulated in CRC tissues compared with normal tissue margins. ${ }^{33}$ It has also been reported that NFATC 2 regulates cyclin-dependent kinase 4 by directly binding its promoter ${ }^{12}$ and mouse with 
A
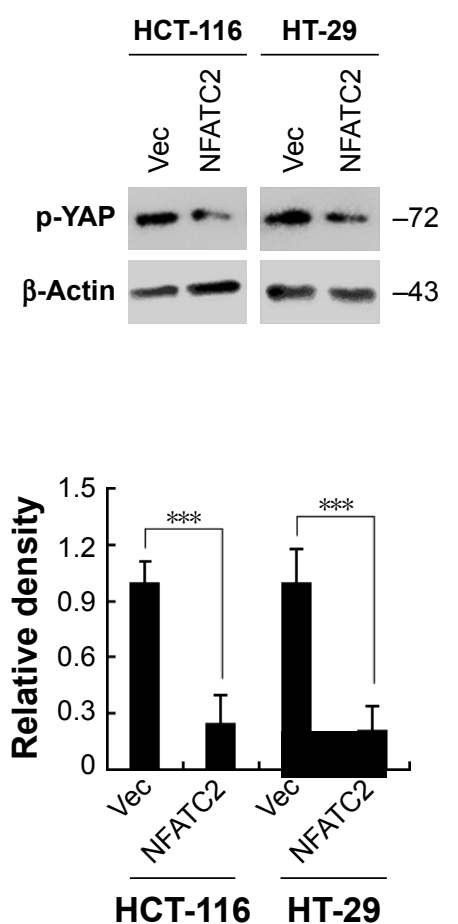

B

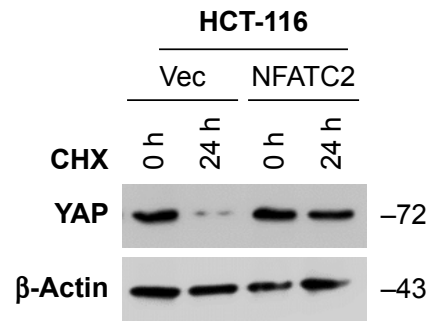

C

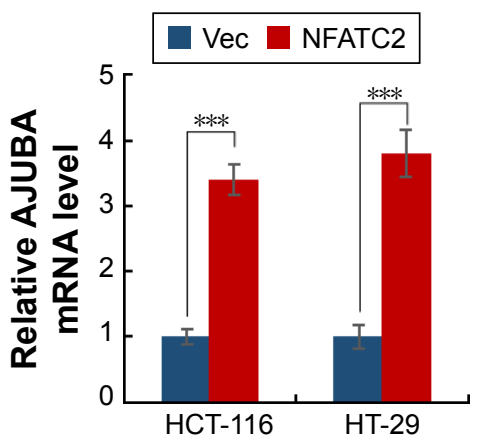

Figure 5 NFATC2 activates Hippo/YAP signaling by upregulation of AJUBA. (A) Western blot analysis of phosphorylated YAP in NFATC2-overexpressing and control HCT-I 16 and HT-29 cells. (B) Western blot analysis of YAP in NFATC2-overexpressing and control HCT-II6 and HT-29 cells pretreated with cycloheximide for the inhibition of new protein synthesis. (C) qRT-PCR analysis of AJUBA in NFATC2-overexpressing and control HCT-II6 and HT-29 cells. (D) qRT-PCR analysis of CTGF and GLI2 in AJUBA-knockdown NFATC2-overexpressing and control HCT-I I6 and HT-29 cells. Data are represented as mean \pm SD; $* * * P<0.001$; two-tailed Student's $t$-test. Abbreviations: CRC, colorectal cancer; NFATC2, nuclear factor of activated T-cells, cytoplasmic 2; CRC-SCs, colorectal cancer stem cells; qRT-PCR, quantitative real-time polymerase chain reaction.

NFATC2 knockout exhibits altered cell cycle control. ${ }^{13}$ In addition, NFATC2 activates the $\mathrm{mdm} 2$ oncogene ${ }^{14}$ and enhances the metastasis of breast cancer cell by upregulation of cyclooxygenase- 2 and prostaglandins. ${ }^{15}$ Moreover, in activated peripheral blood lymphocytes, NFATC2 induces telomerase reverse transcriptase.$^{16}$ Furthermore, overexpression of NFATC2 activates c-Myc. ${ }^{17}$ In our study, we first reported that NFATC2 is upregulated in CRC-SCs (Figure 1). We subsequently showed that overexpression of NFATC2 promotes the stem cell-like properties of CRC-SCs (Figure 2). Conversely, knockdown of NFATC2 attenuates these properties of CRC-SCs (Figure 3), which demonstrated that NFATC2 is critical for the maintenance of CRC-SCs and is a novel therapeutic target of CRC-SCs.

Hippo/YAP axis is an important target for CRC therapy. ${ }^{18,19}$ Elevated YAP expression and the gene signature of high YAP activity have been found in most of the CRC patients and $\mathrm{Apc}^{\mathrm{Min} /+}$ mouse model. ${ }^{34,35}$ YAP knockdown suppresses adenoma caused by loss of APC in mouse intestine. ${ }^{36}$ YAP depletion inhibits the tumorigenicity of human CRC cell lines. ${ }^{37}$ More importantly, YAP has been shown to be dispensable for normal tissue homeostasis, thus limiting the probability of side effects from YAP inhibition. ${ }^{38}$ In this study, we found that NFATC2 upregulates the target genes of Hippo/YAP signaling pathway in CRC cells (Figure S3C and D). We then silenced YAP in NFATC2-overexpressing primary CRC cells and found that YAP depletion abolished the stimulatory effect of NFATC 2 on self-renewal, expression of stem cell markers, and tumorigenicity of CRC-SCs (Figure 4). Furthermore, we showed that AJUBA mediates the stimulatory effect of NFATC2 on YAP activity (Figure 5). These results demonstrated that NFATC2 promotes the stemness of CRC-SCs mainly by suppression of Hippo signaling and thus suggested that YAP inhibitor could be an effective therapeutic strategy for human CRC caused by aberrant activation of NFATC2.

\section{Conclusion}

In summary, we demonstrate that NFATC2 promotes the stemness of CRC-SCs by AJUBA-mediated activation of Hippo/YAP signaling pathway. Our finding reveals that 


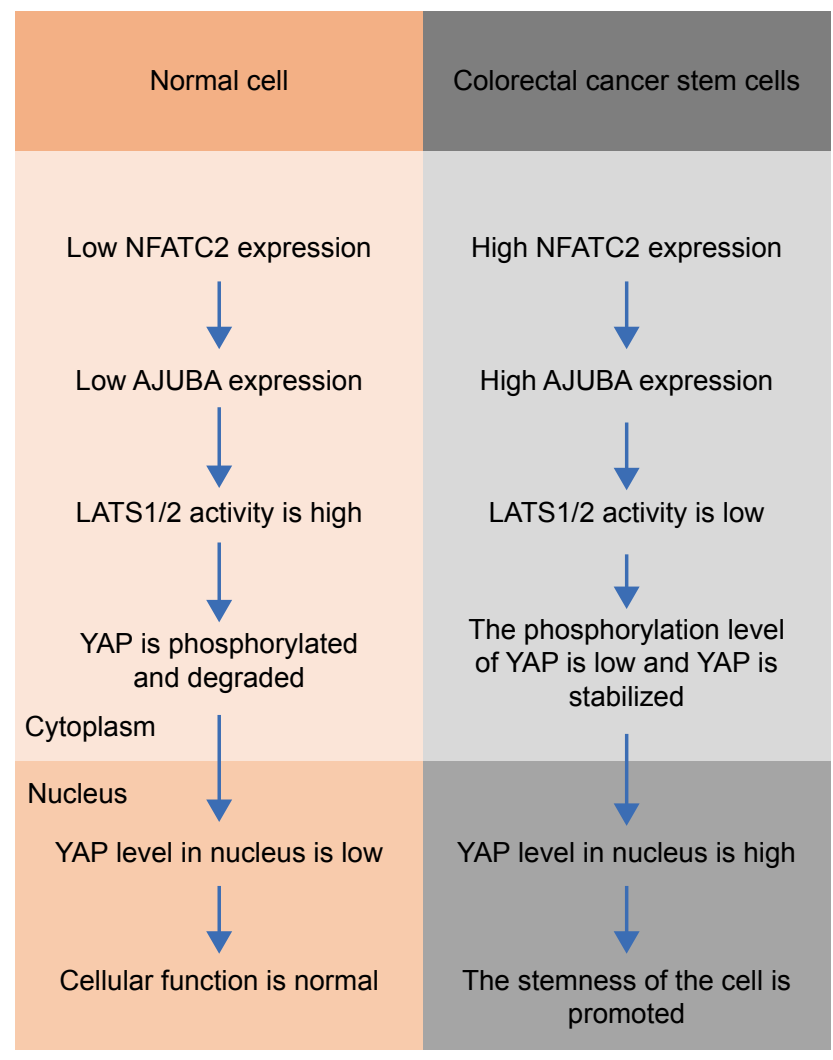

Figure 6 Schematic diagram of NFATC2 promoting the stemness of CRC-SCs. In colorectal cancer stem cells (right), the expression of NFATC2 is upregulated, compared to normal cells (left). Upregulated NFATC2 increases the expression leve of AJUBA, and thus stabilizes YAP through AJUBA-mediated inhibition of LASTI/2, which phosphorylates YAP and promotes $\beta$-TRCP-dependent degradation of YAP. Stabilized YAP translocates into nucleus, activates the transcription of its target genes, and thereby promoting the stemness in colorectal cancer stem cells. Abbreviations: NFATC2, nuclearfactor of activated T-cells, cytoplasmic 2; CRC-SCs, colorectal cancer stem cells; $\beta$-TRCP, $\beta$-transducin repeat-containing E3 ubiquitin protein ligase.

NFATC2 is a novel therapeutic target for CRC-SCs and suggests that YAP inhibitor could be an effective therapeutic strategy for human CRC caused by aberrant activation of NFATC2.

\section{Acknowledgments}

This work was supported by National Natural Science Foundation of China (81572080) and the Special Project for Social Livelihood and Technological Innovation of Chongqing (cstc2016shmszx130043).

\section{Disclosure}

The authors report no conflicts of interest in this work.

\section{References}

1. Siegel RL, Miller KD, Fedewa SA, et al. Colorectal cancer statistics, 2017. CA Cancer J Clin. 2017;67(3):177-193.
2. Findlay VJ, Wang C, Watson DK, Camp ER. Epithelial-to-mesenchymal transition and the cancer stem cell phenotype: insights from cancer biology with therapeutic implications for colorectal cancer. Cancer Gene Ther. 2014;21(5):181-187.

3. Mancini R, Noto A, Pisanu ME, De Vitis C, Maugeri-Saccà M, Ciliberto G. Metabolic features of cancer stem cells: the emerging role of lipid metabolism. Oncogene. 2018;37(18):2367-2378.

4. Dean M, Fojo T, Bates S. Tumour stem cells and drug resistance. Nat Rev Cancer. 2005;5(4):275-284.

5. Wang ZA, Shen MM. Revisiting the concept of cancer stem cells in prostate cancer. Oncogene. 2011;30(11):1261-1271.

6. Ng A, Barker N. Ovary and fimbrial stem cells: biology, niche and cancer origins. Nat Rev Mol Cell Biol. 2015;16(10):625-638.

7. Smalley M, Ashworth A. Stem cells and breast cancer: a field in transit. Nat Rev Cancer. 2003;3(11):832-844.

8. Vaiopoulos AG, Kostakis ID, Koutsilieris M, Papavassiliou AG. Colorectal cancer stem cells. Stem Cells. 2012;30(3):363-371.

9. Zhou BB, Zhang H, Damelin M, Geles KG, Grindley JC, Dirks PB. Tumour-initiating cells: challenges and opportunities for anticancer drug discovery. Nat Rev Drug Discov. 2009;8(10):806-823.

10. Visvader JE. Cells of origin in cancer. Nature. 2011;469(7330): 314-322.

11. Macian F. NFAT proteins: key regulators of T-cell development and function. Nat Rev Immunol. 2005;5(6):472-484.

12. Mognol GP, Carneiro FR, Robbs BK, Faget DV, Viola JP. Cell cycle and apoptosis regulation by NFAT transcription factors: new roles for an old player. Cell Death Dis. 2016;7:e2199.

13. Hodge MR, Ranger AM, Charles de la Brousse F, Hoey T, Grusby MJ, Glimcher LH. Hyperproliferation and dysregulation of IL-4 expression in NF-ATp-deficient mice. Immunity. 1996;4(4):397-405.

14. Zhang X, Zhang Z, Cheng J, et al. Transcription factor NFAT1 activates the $\mathrm{mdm} 2$ oncogene independent of p53. J Biol Chem. 2012; 287(36):30468-30476.

15. Duque J, Fresno M, Iñiguez MA. Expression and function of the nuclear factor of activated $\mathrm{T}$ cells in colon carcinoma cells: involvement in the regulation of cyclooxygenase-2. J Biol Chem. 2005;280(10):8686-8693.

16. Chebel A, Rouault JP, Urbanowicz I, et al. Transcriptional activation of hTERT, the human telomerase reverse transcriptase, by nuclear factor of activated T cells. J Biol Chem. 2009;284(51):35725-35734.

17. Müller MR, Rao A. NFAT, immunity and cancer: a transcription factor comes of age. Nat Rev Immunol. 2010;10(9):645-656.

18. Hong AW, Meng Z, Guan KL. The Hippo pathway in intestinal regeneration and disease. Nat Rev Gastroenterol Hepatol. 2016;13(6):324-337.

19. Pan D. The Hippo signaling pathway in development and cancer. Dev Cell. 2010;19(4):491-505.

20. Yu FX, Meng Z, Plouffe SW, Guan KL. Hippo pathway regulation of gastrointestinal tissues. Annu Rev Physiol. 2015;77:201-227.

21. Hong W, Guan KL. The YAP and TAZ transcription co-activators: key downstream effectors of the mammalian Hippo pathway. Semin Cell Dev Biol. 2012;23(7):785-793.

22. National Research Council. Guide for the Care and Use of Laboratory Animals. 8th ed.; Washington, DC: National Acadamies Press; 2011.

23. Shaheen S, Ahmed M, Lorenzi F, Nateri AS. Spheroid-formation (colonosphere) assay for in vitro assessment and expansion of stem cells in colon cancer. Stem Cell Rev. 2016;12(4):492-499.

24. Cammareri P, Lombardo Y, Francipane MG, Bonventre S, Todaro M, Stassi G. Isolation and culture of colon cancer stem cells. Methods Cell Biol. 2008;86:311-324.

25. Prasetyanti PR, Zimberlin C, De Sousa E, Melo F, Medema JP. Isolation and propagation of colon cancer stem cells. Methods Mol Biol. 2013;1035:247-259.

26. Dotse E, Bian Y. Isolation of colorectal cancer stem-like cells. Cytotechnology. 2016;68(4):609-619.

27. Fang DD, Kim YJ, Lee CN, et al. Expansion of CD133(+) colon cancer cultures retaining stem cell properties to enable cancer stem cell target discovery. Br J Cancer. 2010;102(8):1265-1275. 
28. Du L, Wang H, He L, et al. CD44 is of functional importance for colorectal cancer stem cells. Clin Cancer Research. 2008;14(21):6751-6760.

29. Zhan T, Rindtorff N, Boutros M. Wnt signaling in cancer. Oncogene. 2017;36(11):1461-1473.

30. Jagannathan R, Schimizzi GV, Zhang K, et al. AJUBA LIM proteins limit hippo activity in proliferating cells by sequestering the Hippo core kinase complex in the cytosol. Mol Cell Biol. 2016;36(20): 2526-2542.

31. Kreso A, Dick JE. Evolution of the cancer stem cell model. Cell Stem Cell. 2014;14(3):275-291.

32. Clevers $H$. The cancer stem cell: premises, promises and challenges. Nat Med. 2011;17(3):313-319.

33. Zafari V, Hashemzadeh $\mathrm{S}$, Hosseinpour Feizi M, et al. mRNA expression of nuclear factor of activated T-cells, cytoplasmic 2 (NFATc2) and peroxisome proliferator-activated receptor gamma (PPARG) transcription factors in colorectal carcinoma. Bosn J Basic Med Sci. 2017; 17(3):255-261.
34. Harvey KF, Zhang X, Thomas DM. The Hippo pathway and human cancer. Nat Rev Cancer. 2013;13(4):246-257.

35. Overholtzer M, Zhang J, Smolen GA, et al. Transforming properties of YAP, a candidate oncogene on the chromosome 11q22 amplicon. Proc Natl Acad Sci U S A. 2006;103(33):12405-12410.

36. Cai J, Maitra A, Anders RA, Taketo MM, Pan D. $\beta$-Catenin destruction complex-independent regulation of Hippo-YAP signaling by APC in intestinal tumorigenesis. Genes Dev. 2015;29(14): 1493-1506.

37. Gregorieff A, Liu Y, Inanlou MR, Khomchuk Y, Wrana JL. Yapdependent reprogramming of $\operatorname{Lgr} 5(+)$ stem cells drives intestinal regeneration and cancer. Nature. 2015;526(7575):715-718.

38. Bai H, Zhang N, Xu Y, et al. Yes-associated protein regulates the hepatic response after bile duct ligation. Hepatology. 2012;56(3): 1097-1107. 


\section{Supplementary materials}

Table SI Primer sequences used in this study

\begin{tabular}{|c|c|c|c|}
\hline \multicolumn{4}{|c|}{ Reverse-transcription PCR } \\
\hline Genes & \multicolumn{3}{|l|}{ Primers } \\
\hline \multirow[t]{2}{*}{ NFATC2 } & \multicolumn{3}{|l|}{ F: 5'-ATGCAGAGAGAGGCTGCGTTCAGA-3' } \\
\hline & \multicolumn{3}{|l|}{ R: 5'-TCATAATATGTTTTGTATCCAG-3' } \\
\hline \multicolumn{4}{|c|}{ Quantitative real-time reverse-transcription PCR } \\
\hline \multirow[t]{2}{*}{ CTGF } & F: 5'-CAGCATGGACGTTCGTCTG-3' & \multirow[t]{2}{*}{ GLI2 } & F: 5'-CTGCCTCCGAGAAGCAAGAAG-3' \\
\hline & R: 5'-AACCACGGTTTGGTCCTTGG-3' & & R: 5'-GCATGGAATGGTGGCAAGAG-3' \\
\hline \multirow[t]{2}{*}{ YAP } & F: 5'-TAGCCCTGCGTAGCCAGTTA-3' & \multirow[t]{2}{*}{ CD44 } & F: 5'-AACTAATGCCACCAAGG-3' \\
\hline & R: 5'-TCATGCTTAGTCCACTGTCTGT-3' & & R: 5'-GAAGACGTTTCTTGGCCTGA-3' \\
\hline \multirow[t]{2}{*}{ AJUBA } & F: 5'-ATGGGGAAGTCCTATCATCCAG-3' & \multirow[t]{2}{*}{ CDI33 } & F: 5'-GGCCCAGTACAACACTACCAA- $3^{\prime}$ \\
\hline & R: 5'-TGGTAGTCGGTGACACAGTAT-3' & & R: 5'-ATTCCGCCTCCTAGCACTGAA-3' \\
\hline \multirow[t]{2}{*}{ NFATC2 } & F: 5'-CGATTCGGAGAGCCGGATAG-3' & \multirow[t]{2}{*}{ GAPDH } & F: 5'-GGCTGGGCAAGGTCATCC-3' \\
\hline & R: 5'-TGGGACGGAGTGATCTCGAT-3' & & R: 5'-TCCACCACCCTGTTGCTGTA-3' \\
\hline
\end{tabular}

Table S2 shRNA sequences used in this study

\begin{tabular}{ll}
\hline shNFATC2\#I & F: 5'-TGCTGTTGACAGTGAGCGAGCCCTGCGTCTCGCCCAATAATAGTGAAGCCACAGATGTATT \\
shNFATC2\#2 & ATTGGGGAGACGCAGGGCGTGCCTACTGCCTCGGA-3' \\
& F: 5'-TGCTGTTGACAGTGAGCGCGACCACAGATGGACAGCAAATTAGTGAAGCCACAGATGTAAT \\
shYAP\#I & TTGCTGTCCATCTGTGGTCTTGCCTACTGCCTCGGA-3' \\
& F: 5'-TGCTGTTGACAGTGAGCGCGGAACTATTTGAACAGCTGAATAGTGAAGCCACAGATGTATT \\
shYAP\#2 & CAGCTGTTCAAATAGTTCCTTGCCTACTGCCTCGGA-3' \\
& F: 5'-TGCTGTTGACAGTGAGCGCGCCCAATCAACTCATTCAGAATAGTGAAGCCACAGATGTATT \\
shAJUBA\#I & CTGAATGAGTTGATTGGGCTTGCCTACTGCCTCGGA-3' \\
shAJUBA\#2 & F: 5'-TGCTGTTGACAGTGAGCGACCAAAGATCATGATATTCCAATAGTGAAGCCACAGATGTATTG \\
sh-con & GAATATCATGATCTTTGGGTGCTACTGCCTCGGA-3' \\
& F: 5'-TGCTGTTGACAGTGAGCGAGCATATTCAGTTGACTTACAATAGTGAAGCCACAGATGTATTG \\
& TAAGTCAACTGAATATGCCTGCCTACTGCCTCGGA-3' \\
& F: 5'-CCGGAAACTACCGTTGTTATAGGTGTTCAAGAGACACCTATAACAACGGTAGTTTTTTTTG-3'
\end{tabular}

Table S3 Antibodies used in this study

\begin{tabular}{|c|c|c|c|c|c|c|}
\hline Antigen & Application & Antibody & Conjugated with & Supplier & Catalog \# & Dilution \\
\hline NFATC2 & IB & Rabbit monoclonal & & CST & \#4389 & 2,000 \\
\hline$\beta$-Actin & IB & Mouse monoclonal & & Abcam & Ab6276 & 1,000 \\
\hline CD44 & IB & Mouse monoclonal & & Thermo Fisher Scientific & MS668P0 & 500 \\
\hline CDI33 & IB & Mouse monoclonal & & Miltenyi Biotec & 130-092-395 & 100 \\
\hline CDI33 & FC & Mouse $\lg G 2 b, \kappa$ & FITC & Miltenyi Biotec & $130-104-322$ & According to \\
\hline CD44 & FC & Mouse IgG2b, $\kappa$ & PE & BD Pharmingen & 555479 & the manual \\
\hline YAP & $\mathrm{IB}, \mathrm{IHC}$ & Rabbit polyclonal & & CST & $\# 14074$ & $\begin{array}{l}\text { IB: I,000 } \\
\text { IHC: } 500\end{array}$ \\
\hline AJUBA & IB & Rabbit monoclonal & & CST & \#34648 & 1,000 \\
\hline Rabbit IgG (H+L) & IB & Goat polyclonal & Horseradish peroxidase & CST & 7074 & 1,000 \\
\hline Mouse IgG $(\mathrm{H}+\mathrm{L})$ & IB & Hourse polyclonal & Horseradish peroxidase & CST & 7076 & 1,000 \\
\hline Goat $\lg G(H+L)$ & $\mathrm{IHC}$ & Donkey polyclonal & DyLight $^{\circledR} 488$ & Abcam & Ab985I4 & 500 \\
\hline Goat $\lg G(\mathrm{H}+\mathrm{L})$ & $\mathrm{IHC}$ & Donkey polyclonal & Alexa Fluor ${ }^{\circledR} 555$ & Abcam & $\mathrm{AbI} 50 \mathrm{I} 34$ & 500 \\
\hline Rabbit IgG $(\mathrm{H}+\mathrm{L})$ & $\mathrm{IHC}$ & Donkey polyclonal & Alexa Fluor ${ }^{\circledR} 488$ & Abcam & Abl50073 & 500 \\
\hline
\end{tabular}

Abbreviations: IB, immunoblotting; FC, flow cytometry; IHC, immunohistochemistry. 


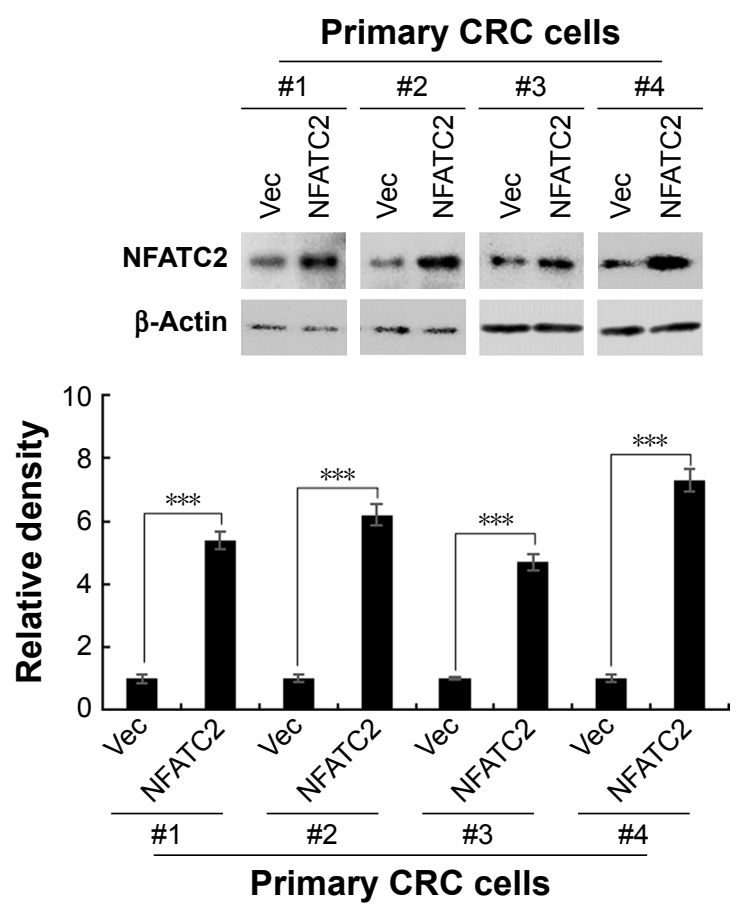

Figure SI Characterization of NFATC2-overexpressing primary CRC cells. Bottom panel: statistical analysis of the blots. Data are represented as mean \pm SD; $* * * P<0.00$ I; two-tailed Student's $t$-test.

Abbreviations: CRC, colorectal cancer; NFATC2, nuclear factor of activated T-cells, cytoplasmic 2.

Primary CRC cells
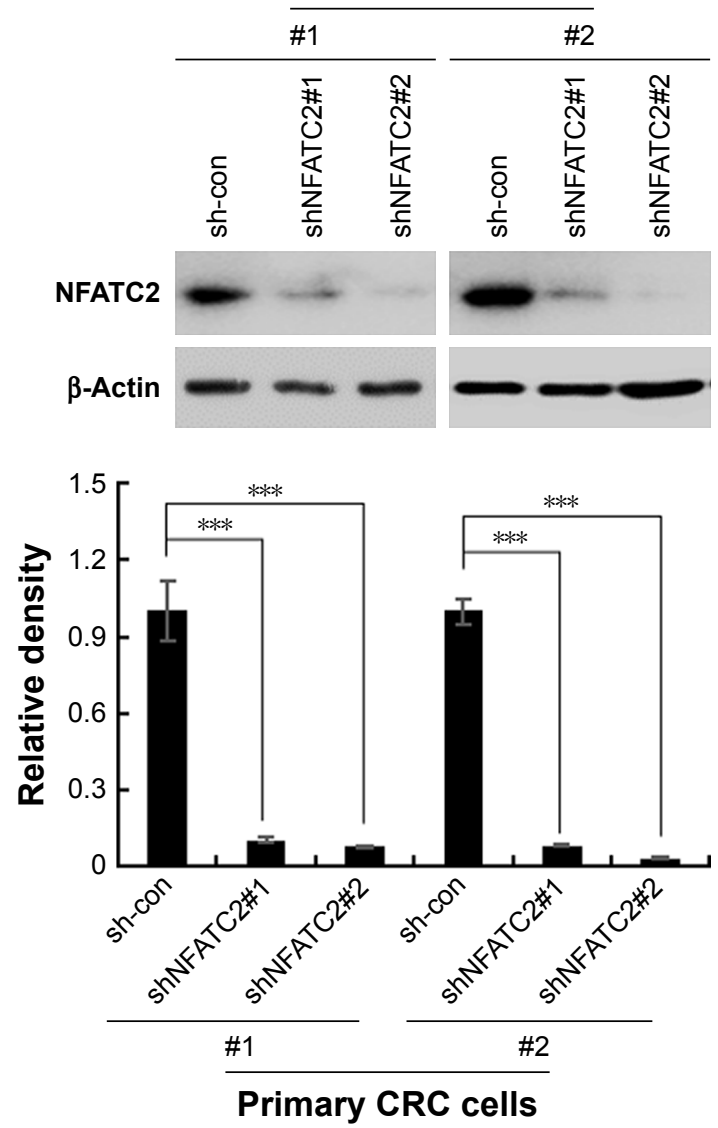

Figure S2 Characterization of NFATC2-knockdown primary CRC cells.

Note: Data are represented as mean $\pm S D$; $* * * P<0.00$ I; two-tailed Student's $t$-test.

Abbreviations: CRC, colorectal cancer; NFATC2, nuclear factor of activated T-cells, cytoplasmic 2. 
A
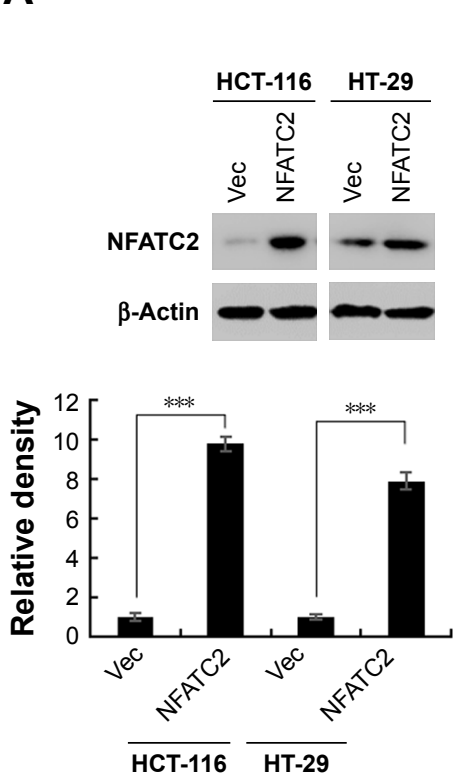

D

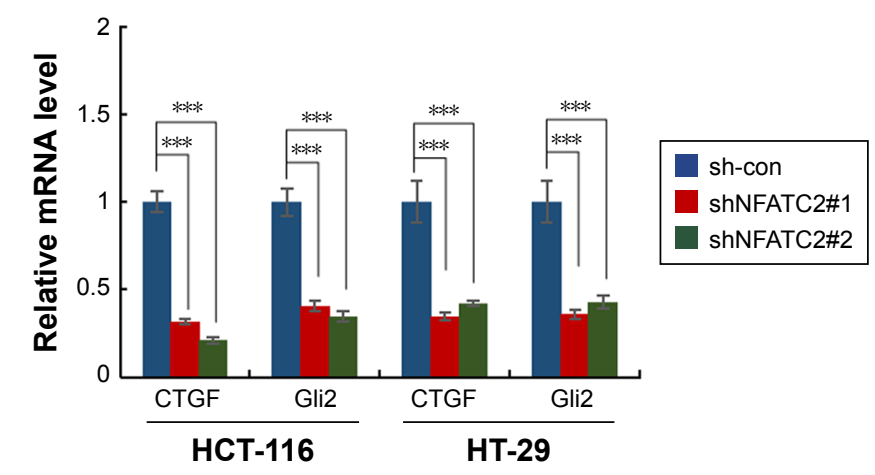

B
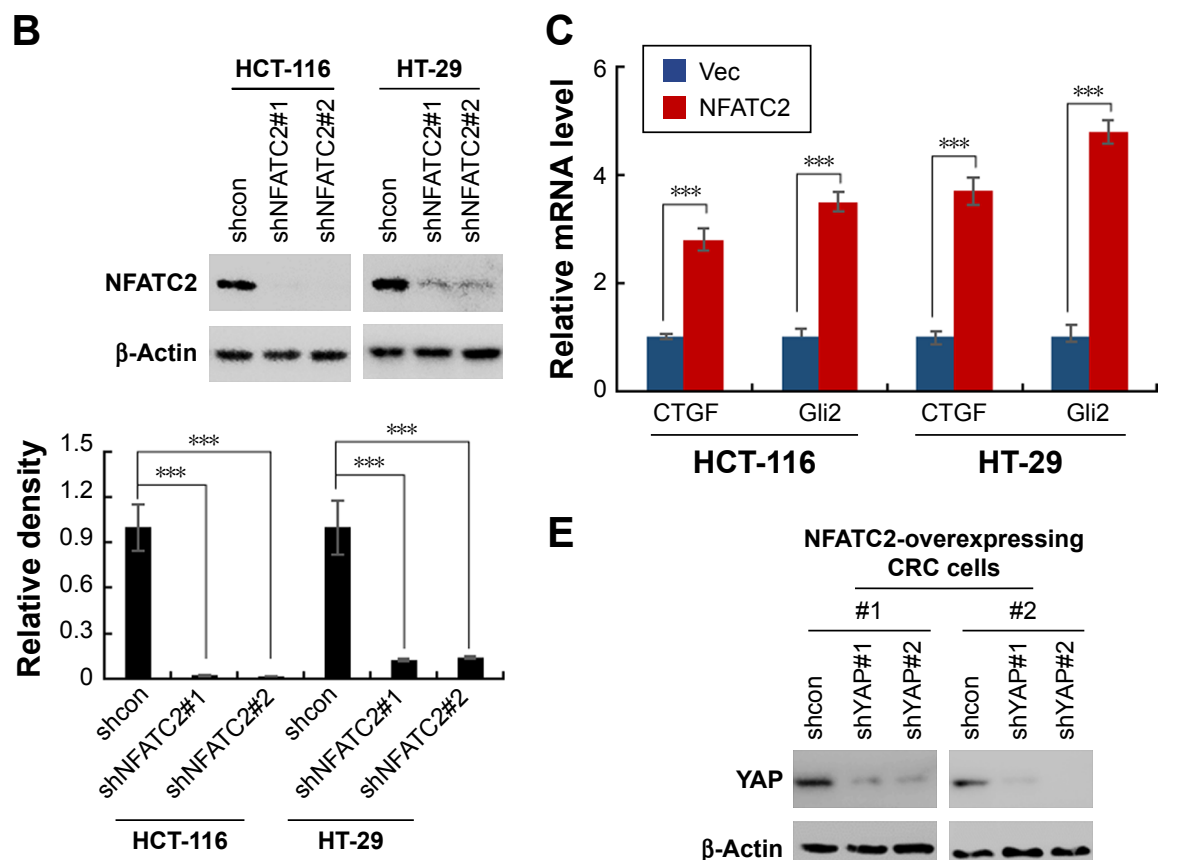

E

NFATC2-overexpressing CRC cells
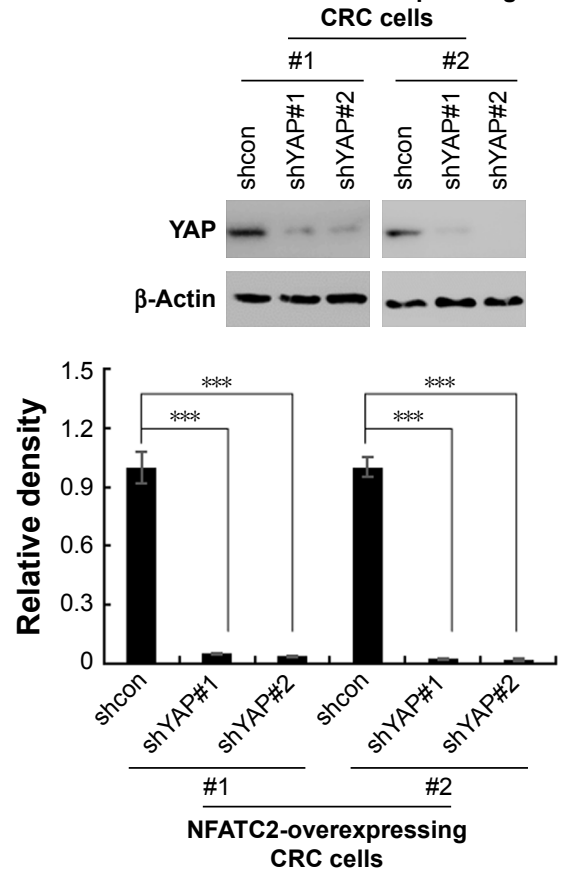

Figure S3 NFATC2 regulates Hippo/YAP signaling pathway. (A) Characterization of NFATC2-overexpressing HCT-1 16 and HT-29 cells. (B) Characterization of NFATC2knockdown HCT-1 16 and HT-29 cells. (C) qRT-PCR analysis of CTGF and Gli2 in NFATC2-overexpressing HCT-1 16 and HT-29 cells. (D) qRT-PCR analysis of CTGF and Gli2 in NFATC2-knockdown HCT-I I6 and HT-29 cells. (E) Characterization of YAP-knockdown NFATC2-overexpressing CRC cells.

Note: Data are represented as mean $\pm S D$; $* * * P<0.001$; two-tailed Student's $t$-test.

Abbreviations: CRC, colorectal cancer; NFATC2, nuclear factor of activated T-cells, cytoplasmic 2; qRT-PCR, quantitative real-time polymerase chain reaction. 
A

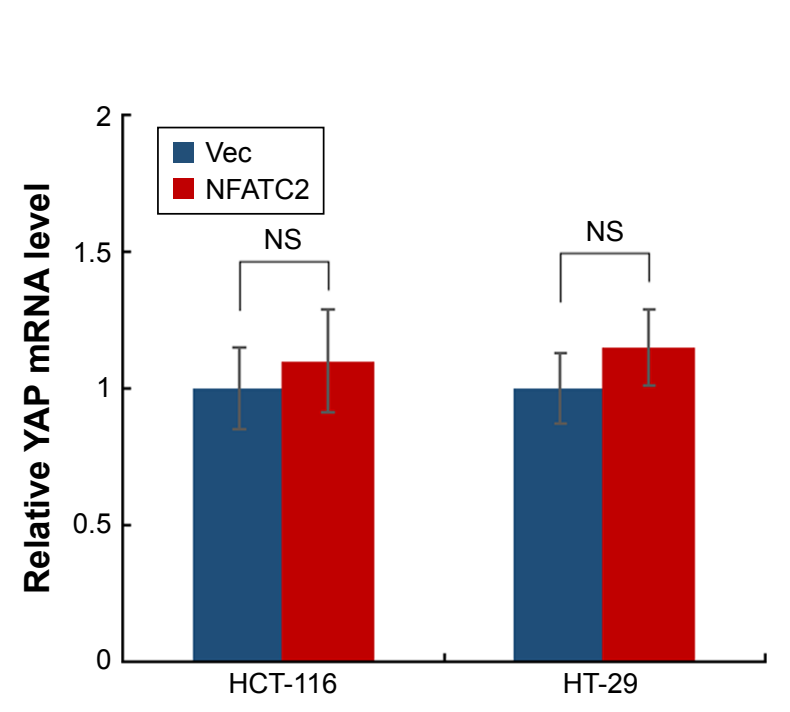

B

HCT-116-NFATC2 HT-29-NFATC2
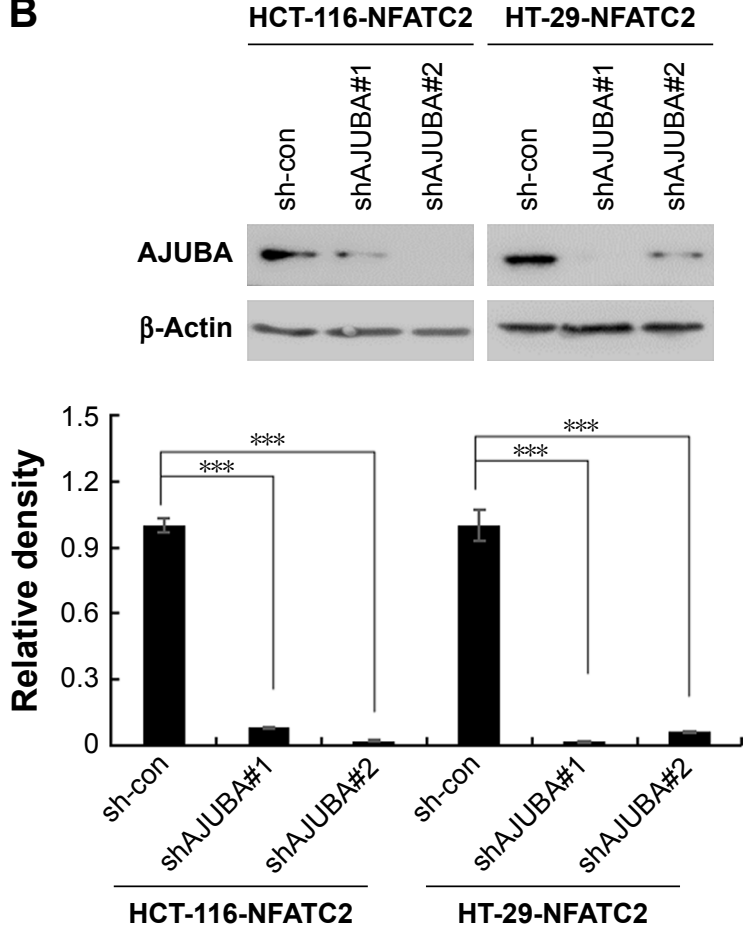

Figure S4 NFATC2 regulates Hippo/YAP signaling pathway through AJUBA. (A) qRT-PCR analysis of YAP in NFATC2-overexpressing HCT-II6, HT-29 cells and their control cells. (B) Characterization of AJUBA-knockdown NFATC2-overexpressing HCT-1 16 and HT-29 cells.

Note: Data are represented as mean $\pm \mathrm{SD}$; $* * * P<0.001$; two-tailed Student's $t$-test.

Abbreviations: NFATC2, nuclear factor of activated T-cells, cytoplasmic 2; qRT-PCR, quantitative real-time polymerase chain reaction.

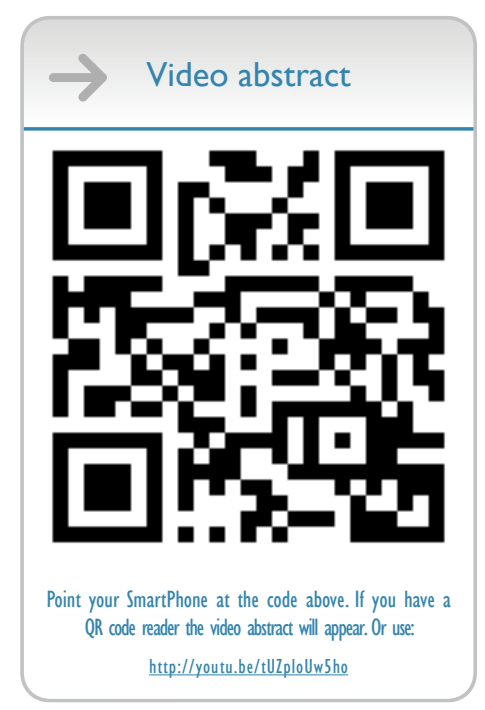

OncoTargets and Therapy

Dovepress

\section{Publish your work in this journal}

OncoTargets and Therapy is an international, peer-reviewed, open access journal focusing on the pathological basis of all cancers, potential targets for therapy and treatment protocols employed to improve the management of cancer patients. The journal also focuses on the impact of management programs and new therapeutic agents and protocols on

patient perspectives such as quality of life, adherence and satisfaction. The manuscript management system is completely online and includes a very quick and fair peer-review system, which is all easy to use. Visit http://www.dovepress.com/testimonials.php to read real quotes from published authors. 